\title{
Kinetic Friction due to Ohm's Law Heating
}

\author{
J. B. Sokoloff, Physics Department and \\ Center for Interdisciplinary Research on complex Systems, \\ Northeastern University, Boston, Massachusetts 02115
}




\title{
Kinetic Friction due to Ohm's Law Heating
}

\author{
J. B. Sokoloff, Physics Department and \\ Center for Interdisciplinary Research on complex Systems, \\ Northeastern University, Boston, Massachusetts 02115
}

\begin{abstract}
Using both a recent calculation by Bruch of the damping of the motion of a monolayer nitrogen film oscillating harmonically on a metallic surface due to Ohm's law heating and a Thomas-Fermi approximation treatment of the Ohm's law heating mechanism, which accounts for the nonzero thickness of the surface region of a metal, it is argued that this mechanism for friction is able to account for recent measurements of the drop in the friction for a nitrogen film sliding over a lead substrate as it goes below its superconducting transition temperature. Bruch's calculation is also made more transparent by re-doing the calculation for a film sliding at constant speed, instead of oscillating. Using this treatment, it is easily shown that Bruch's calculation is equivalent to integrating Boyer's solution of the problem of a charge sliding over a metallic surface over the charge density of the monolayer nitrogen film.
\end{abstract}

\section{Introduction}


Motivated by attempts[1-3] to explain a recent quartz crystal microbalance experiment[4] which shows a rapid drop in the friction of a film of nitrogen molecules sliding on a lead substrate, on dropping below the superconducting transition temperature $T_{c}$ of the substrate, Bruch has recently done a calculation of the electronic contribution to the friction for a monolayer nitrogen film executing simple harmonic motion on a metallic substrate[5]. In contrast to calculations of a single molecule moving on the substrate[6,8], which require that the molecule possess a larger dipole moment or charge than is generally accepted for adsorbed molecules, in order to explain the experimental results of Ref. 4 [1-3], Bruch's results suggest that the field due to the quadrupole moment of the nitrogen molecule can explain the microbalance experiments[5] if the molecules form a monolayer film. The reason for this is that for a monolayer film the field inside the metallic substrate on which the film is moving falls exponentially to zero over a distance of the order of a lattice constant of the film below the surface of the metal. Since the distance over which the field is nonzero is much smaller than a mean free path, Bruch pointed out that the problem must be treated in the anomalous skin effect regime[7], in which only those electrons with velocities nearly parallel to the film remain in this region for a sufficient length of time to be significantly accelerated by the electric field. Consequently, only a fraction 
$(G \ell)^{-1}$ of the electrons (where $\mathrm{G}$ is the magnitude of a typical reciprocal lattice vector and $\ell$ is the mean free path) can be accelerated by the field. This results in an enhancement of the effective resistivity of the skin depth region, which leads to an enhancement of the rate of dissipation and the contribution to the kinetic friction due to Ohm's law heating.

In addition to the Ohm's law heating mechanism for electronic friction put forward in Refs. 1 and 2 as a possible way of explaining the experimental results reported in Ref. 4, there is another mechanism for electronic friction (actually, the most commonly suggested mechanism for this phenomenon) which is due to the creation of electron-hole pairs of nonzero energy[9-12]. The physical difference between these two mechanisms can be understood as follows: In the electronhole pair mechanism for electronic friction, the energy loss due to friction is ascribed to the energy needed to create the electron-hole pairs. In contrast, in the Ohm's law heating mechanism considered in references 1,2 and 5 and in this article, the energy loss need not be due to the energy cost necessary to create electron hole pairs. In this mechanism, if we consider the case in which the film is slid along at constant speed (e.g., by an applied force), the electric field resulting from the sliding film results in a screening charge near the surface of the metal, which is dragged along with the film. This results in an electric 
current. Let us first consider only elastic scattering of the electrons (the dominant contribution to the resistivity well below the Debye temperature). During the sliding, electrons get scattered elastically by impurities and other defects. This would result in a reduction in the drift velocity, and hence the electric current, except that we force the current to remain constant by forcing the film to move at constant speed. In order to maintain the current, the electric field acting on the conduction electrons due to the film must accelerate them in order to maintain the drift velocity. The work done by this field is identified with the contribution to the dissipation produced by Ohm's law heating. In the Ohm's law heating mechanism considering only elastic scattering of the electrons, the electron-hole pairs resulting from the scattering of the electrons by defects in the substrate have zero energy because the scattering is elastic. In addition to the contribution due to elastic scattering, there is also inelastic scattering of the electrons by phonons, which results in an additional energy loss from energy transferred from the electrons to the phonons. Below $T_{c}$, the above scattering mechanisms do not occur for the superconducting electrons because of the gap in their excitation spectrum. The screening charge will be transported entirely by the superconducting electrons because they can flow without electrical resistance, and hence are able to short circuit the current due to the normal electrons. 
Since the superconducting electrons are not scattered, the Ohm's law heating mechanism for dissipation (and hence kinetic friction) described above does not operate.

Persson has argued that the electron-hole pair mechanism should dominate over the Ohm's law heating mechanism by three orders of magnitude for a charged ion moving above the surface of a metallic substrate[13]. Since this mechanism depends on the density of normal electrons, which does not drop rapidly on falling below the superconducting transition temperature, however, it cannot account for the experimental result reported in Ref. 4. The calculation of the Ohm's law heating contribution of the friction, including the anomalous skin effect conductivity, as suggested by Bruch[5], however, can be of the same order of magnitude as the electron-hole contribution to the friction or greater. Since the resistivity drops to zero over a relatively small temperature range on dropping below $T_{c}$, the latter mechanism for electronic friction drops rapidly in much the same way as in Ref. 4, and, as we shall see, it is large enough to explain the experimental observations.

In Bruch's treatment, the simple harmonic motion of the film results in an electric field with many harmonics of the frequency of the film's oscillations, which is an unnecessary complication in his treatment. In contrast, if one considers the 
film to be sliding over the substrate at constant speed, the field will possess only a single harmonic of the "washboard frequency," which is the characteristic frequency in this formulation of the problem. In section II, Bruch's calculation is reformulated by assuming that the film slides at constant velocity, rather than executing simple harmonic motion. It is expected that this will give the same value for the friction, and it simplifies the calculation. Furthermore, doing the calculation in this way makes it possible to verify that the force of friction acting on the sliding film calculated from the Ohmic heating inside the metallic substrate is equal to the friction found by calculating the force on the film due to its image field acting back on it, as is required by energy conservation. (This is not possible in Bruch's treatment because when averaged over the oscillation period of the film, the force of friction averages to zero.) It is argued in section III, that this version of Bruch's treatment is identical to Boyer's treatment[6], which is identical to the treatment of the problem due to Tomassone and Widom[8], used in Refs. 1 and 2. Since Ref. 13 stresses the necessity of taking into account the nonzero thickness of the surface region, and since, Bruch's work is a classical treatment of the surface of a metal (i.e., one which assumes the surface region has zero thickness), in section IV, a calculation is presented which takes into account the fact that the surface region, when treated quantum mechanically, has a nonzero 
thickness, in contrast to the zero thickness that it has in treatments of this problem using classical electrodynamics [1-3,5-8].

\section{Bruch's Idea Applied to a Uniformly Sliding Film}

Let us take the region with $z<0$ to be occupied by the metal, with the region with $z>0$ occupied by free space. Bruch [5] writes the z-component of the electric field in terms of its Fourier transform on the $\mathrm{x}$ and $\mathrm{y}$ coordinates, $E(\mathbf{G}, z)$,

$$
E_{z}(\mathbf{r})=\sum_{\mathbf{G}} E(\mathbf{G}, z) e^{i \mathbf{G} \cdot \mathbf{r}}
$$

where $\mathbf{G}$ denotes a reciprocal lattice vector of the nitrogen film. Using the requirement that the field have zero divergence (in regions with zero charge density), the field components parallel to the surface are given by

$$
\mathbf{E}_{\|}(\mathbf{r})=\sum_{\mathbf{G}}\left(i \mathbf{G} / G^{2}\right) \partial E(\mathbf{G}, z) / \partial z e^{i \mathbf{G} \cdot \mathbf{r}} .
$$

In regions in which there is no net charge density the current density $\mathbf{J}(\mathbf{r})$ can similarly be expressed in terms of the Fourier transform of its z-component. In Bruch's work, the film is assumed to execute simple harmonic motion of frequency $\Omega$ as a rigid unit, which means that the time dependent fields are obtained by replacing $\mathbf{r}$ by $\mathbf{r}-\mathbf{A} \cos (\Omega t)$, where the vector $\mathbf{A}$ has magnitude equal to the 
amplitude and direction in the direction of motion of the film. When this substitution is made in Eqs. (1) and (2), the time dependence of the fields is a sum of harmonics $K \Omega$, where $\mathrm{K}$ is an integer, with Bessel function coefficients $J_{K}(\mathbf{G} \cdot \mathbf{A})$. In Bruch's solution it is necessary to deal with all of the time Fourier components. In this section, I propose that the inverse slip-time, which Bruch obtains, can be obtained much more easily by considering the damping of a film moving at constant velocity $\mathbf{v}$ instead. For the present treatment, in which the film slides at constant velocity $\mathbf{v}$, we obtain the time dependence simply by replacing $\mathbf{r}$ by $\mathbf{r}-\mathbf{v t}$, which results in a field which contain only a single time Fourier component for each reciprocal lattice vector, with frequency equal to the "washboard frequency," $\mathbf{G} \cdot \mathbf{v}$, and there is no sum over harmonic with Bessel function coefficients. Because of the linear nature of Ohm's law, this method must give the same value for the slip-time as Bruch obtains. Bruch obtains a relationship between the the time and space Fourier transforms of the z-component of the current density inside the film, and the z-component of the time and space Fourier component of the field on the surface of the metal, just inside the metal [Eq. (2.10) in Bruch's paper] by solving the linearized Boltzmann equation simultaneously with Faraday's and Ampere's laws. Because the frequencies involved in this problem are quite small, Bruch solves these equations in the zero frequency limit. 
As a consequence, each time Fourier component of the field and the current density satisfies the same equations (since in the zero frequency limit, the coefficients multiplying the fields in the equations are obviously independent of frequency). Thus, Bruch's solution of Boltzmann's equation with Faraday's and Ampere's law can be equally well applied to the present case of a film sliding at constant velocity, for which there is only one time Fourier component for each reciprocal lattice vector $\mathbf{G}$, e.g., $\mathbf{E}(\mathbf{G}, z)$ for the z-component of the electric field. One obtains for the relationship between the Fourier transforms of the z-component of the current density and field just below the surface of the metal

$$
J\left(\mathbf{G}, z=0^{-}\right)=\sigma_{\mathbf{G}} E\left(\left(\mathbf{G}, z=0^{-}\right),\right.
$$

where $\sigma_{\mathbf{G}}=3 \sigma(1-p) /(4 G \ell)$, where $\ell$ is the mean free path, $\mathrm{p}$ is the fraction of the conduction electrons which are specularly reflected at the surface of the metal at $\mathrm{z}=0$ and $\sigma$ is the Ohm's law electrical conductivity. This is Bruch's Eq. (2.10). Substituting Eq, (3) in the standard boundary condition [14]

$$
J_{z}\left(\mathbf{r}, z=0^{-}\right)=(4 \pi)^{-1} \frac{\partial}{\partial t}\left[E_{z}\left(\mathbf{r}, z=0^{-}\right)-E_{z}\left(\mathbf{r}, z=0^{+}\right)\right]
$$

we obtain

$$
\sum_{\mathbf{G}} \sigma_{\mathbf{G}} E\left(\mathbf{G}, z=0^{-}\right) e^{i \mathbf{G} \cdot \mathbf{r}}=
$$




$$
\begin{gathered}
(4 \pi)^{-1} \partial\left[E_{z}\left(z=0^{-}\right)-E_{z}\left(z=0^{+}\right)\right] / \partial t= \\
i(4 \pi)^{-1} \sum_{\mathbf{G}}(\mathbf{G} \cdot \mathbf{v})\left[E\left(\mathbf{G}, z=0^{-}\right)-E\left(\mathbf{G}, z=0^{+}\right)\right] e^{i \mathbf{G} \cdot \mathbf{r}},
\end{gathered}
$$

where we have used the fact that $\mathrm{E}$ and $\mathrm{J}$ have the time dependence $e^{-i \mathbf{G} \cdot \mathbf{v} t}$ for a film sliding at constant speed, for the reasons given above. Eq. (5) can be written as

$$
\left(1+i \lambda_{\mathbf{G}}\right) E\left(\mathbf{G}, 0^{-}\right)=E\left(\mathbf{G}, 0^{+}\right)=B_{\mathbf{G}} e^{G z}+E_{i}\left(\mathbf{G}, 0^{+}\right)
$$

where $E_{i}(\mathbf{G}, z)$ and $E(\mathbf{G}, z)$ are the Fourier transform of the z component of the contribution to the field from the film in the absence of the substrate and inside the metal, respectively, and $B_{\mathbf{G}} e^{G z}$ is the contribution of the Fourier transform of the $\mathrm{z}$ component of the field outside of the substrate due to the charge density induced by the film. $B_{\mathbf{G}}$ is a constant to be determined by the boundary conditions. The parameter $\lambda_{\mathbf{G}}=4 \pi \sigma_{\mathbf{G}} /(\mathbf{G} \cdot \mathbf{v})$, where $(\mathbf{G} \cdot \mathbf{v})$ is the "washboard" frequency of the film. In addition to Eq. (6), we must require continuity of the component of the field parallel to the surface, which is given by

$$
d E(\mathbf{G}, z) /\left.d z\right|_{z=0^{-}}=\left.\left[d\left(B_{\mathbf{G}} e^{G z}\right) / d z+d E_{i}(\mathbf{G}, z) / d z\right]\right|_{z=0^{+}}
$$

which using the result

$$
E(\mathbf{G}, z)=E\left(\mathbf{G}, z=0^{-}\right) e^{G z}
$$


given in Eq. (A13) of the appendix of Ref. 5 (It follows from the solution of Ampere's and Faraday's law in the zero frequency limit.) gives

$$
-E\left(\mathbf{G}, 0^{+}\right)=B_{\mathbf{G}}-E_{i}\left(\mathbf{G}, 0^{-}\right) .
$$

The solution to Eqs. (6) and (8) is

$$
E\left(\mathbf{G}, 0^{+}\right)=\left[2 /\left(2+i \lambda_{\mathbf{G}}\right)\right] E_{i}\left(\mathbf{G}, 0^{-}\right),
$$

and

$$
B_{\mathbf{G}}=\left[i \lambda_{\mathbf{G}} /\left(2+i \lambda_{\mathbf{G}}\right)\right] E_{i}\left(\mathbf{G}, 0^{-}\right) \approx\left(1+2 i / \lambda_{\mathbf{G}}+\ldots\right) E_{i}\left(\mathbf{G}, 0^{-}\right),
$$

where $E_{i}(\mathbf{G}, Z)$ can be found using

$$
E_{i}(\mathbf{r})=\int d^{3} r^{\prime} \rho(\mathbf{r}) \frac{\mathbf{r}-\mathbf{r}^{\prime}}{\left|\mathbf{r}-\mathbf{r}^{\prime}\right|^{3}}
$$

where $\rho(\mathbf{r})$ is the film's charge density. It can be crudely modeled by three charges along the axis of a molecule $\ell_{\beta}$, in the small $\ell_{\beta}$ limit, two charges of charge $+q$ at the outer edges of the molecule and a charge $-2 q$ at its center. The value of $q$ is chosen such that the resulting quadrupole moment has the experimental value $\theta$. Then, in the unit cell near the origin

$$
\rho(\mathbf{r})=q \delta(z+h) \sum_{\beta=1,2}\left[\delta^{(2)}\left(\mathbf{r}-\rho_{\beta}-\ell_{\beta}\right)+\delta^{(2)}\left(\mathbf{r}-\rho_{\beta}+\ell_{\beta}\right)\right.
$$




$$
\left.-2 \delta^{(2)}\left(\mathbf{r}-\rho_{\beta}\right)\right]
$$

where $\delta^{(2)}$ denotes a two dimensional delta function and $\rho_{\beta}$ denotes the position of a molecule in the unit cell. Writing

$$
\frac{\mathbf{r}-\mathbf{r}^{\prime}}{\left|\mathbf{r}-\mathbf{r}^{\prime}\right|^{3}}=4 \pi(2 \pi)^{-3} \int d^{3} k\left(i \mathbf{k} / k^{2}\right) e^{i \mathbf{k} \cdot\left(\mathbf{r}-\mathbf{r}^{\prime}\right)}
$$

and substituting in Eq. (10), we obtain

$$
E_{i}\left(\mathbf{G}, 0^{-}\right)=-\left(\pi \theta / A_{c}\right) e^{-G h} \sum_{\beta} e^{-i \mathbf{G} \cdot \rho_{\beta}}\left(\mathbf{G} \cdot \hat{\ell}_{\beta}\right)^{2},
$$

where $\rho_{\beta}$ is the location of the $\beta^{\text {th }}$ molecule in the nitrogen film unit cell, $\hat{\ell}_{\beta}$ is the symmetry axis of $\beta^{\text {th }}$ molecule, and $\theta$ is the quadrupole moment of a single molecule. The inverse slip-time found by calculating the force exerted on the film by the image field outside the metal,

$$
\sum_{\mathbf{G}}\left(i \mathbf{G} / G^{2}\right) \partial\left[B_{\mathbf{G}} e^{G z} e^{i \mathbf{G} \cdot \mathbf{r}}\right] / \partial z
$$

is given by $(N M v)^{-1}$ times the force or

$$
(N M v)^{-1} \int d^{3} r \rho(\mathbf{r}) \sum_{\mathbf{G}}\left(i \mathbf{G} / G^{2}\right) \partial\left[B e^{G z} e^{i \mathbf{G} \cdot \mathbf{r}}\right] / \partial z .
$$

From Eqs. (9b) and (13), we obtain for the inverse slip-time

$$
\tau^{-1}=\left(\frac{\theta^{2}}{3(1-p) \sigma A_{c} M v}\right) \mid \sum_{\mathbf{G}} e^{-2 G h}(4 G \ell)(\mathbf{G} \cdot \mathbf{v})(\mathbf{G} / G)
$$




$$
\left|\sum_{\beta}\left(\mathbf{G} \cdot \hat{\ell}_{\beta}\right)^{2} e^{i \mathbf{G} \cdot \rho_{\beta}}\right|^{2} \mid,
$$

which gives

$$
\begin{gathered}
\tau^{-1}=\left(\frac{\theta^{2}}{3(1-p) \sigma A_{c} M}\right) \sum_{\mathbf{G}}(4 G \ell)\left(G_{x}^{2} / G\right) e^{-2 G h} \\
\left|\sum_{\beta}\left(\mathbf{G} \cdot \hat{\ell}_{\beta}\right)^{2} e^{i \mathbf{G} \cdot \rho_{\beta}}\right|^{2},
\end{gathered}
$$

where we have taken $\mathrm{v}$ to be along the $\mathrm{x}$-axis. By energy conservation, this expression for $\tau^{-1}$ must be equal to the value calculated by setting the power loss due to Ohm's law heating equal to $N M v^{2} / \tau$, the rate at which the viscous electronic contribution to the friction force, $N M v / \tau$, does work on the film. Using the method used in this paper of assuming that the film is sliding at a speed v, rather than oscillating (as assumed in Bruch's paper), the Ohm's law heating power is given by

$$
P=2 N A_{c} \sum_{\mathbf{G}} G^{-1}\left|E_{i}\left(\mathbf{G}, 0^{-}\right)\right|^{2}(\mathbf{G} \cdot \mathbf{v}) / \sigma_{\mathbf{G}}
$$

The factor $N A_{c}$ appears because when $\mathrm{P}$ is calculated by integrating $\mathbf{J}(\mathbf{r}) \cdot \mathbf{E}(\mathbf{r})$ over the volume of the metal, the volume integral involves evaluating the integral $\int d^{2} r e^{i\left(\mathbf{G}-\mathbf{G}^{\prime}\right) \cdot \mathbf{r}}=N A_{c} \delta_{\mathbf{G}, \mathbf{G}^{\prime}} \cdot$ We obtain with this proceedure[15], substituting $E_{i}$ from Eq. (12) in Eq. (16), the value for $\tau^{-1}$ obtained in Eq. (15). 


\section{Treatment of Electronic Friction using Boyer's Solution}

Boyer[6] solves the problem of a charge or electric dipole moving above (i.e., outside the metal) and parallel to the surface of the metal. He solves the electrodynamics problem subject to the same boundary conditions as Bruch uses [14], [Eq. (4) above], which for Boyer's problem is written as

$$
J_{z}=\sigma E=(4 \pi)^{-1} \partial E_{z} / \partial t=(4 \pi)^{-1} \mathbf{v} \cdot \nabla E_{z}
$$

When terms of up to first order in $\mathrm{v}$ are kept, we obtain Boyer's result for the

electric field. The force exerted by this field on the moving charge gives the force of friction due to Ohm's law heating in the metal. The force of friction found by the formalism due to Tomassone and Widom[8] gives the same friction and hence is believed to be equivalent to Boyer's calculation.

Boyer[6] finds, in addition to the electrostatic field, a contribution to the electric field above the substrate linear in the velocity $\mathrm{v}$ of a point charge $\mathrm{q}$ sliding above the substrate, given by

$$
\mathbf{E}(\mathbf{r})=-(q v / 2 \pi \sigma) \frac{\partial}{\partial x}\left[\frac{\mathbf{r}-\mathbf{r}^{\prime}}{\left|\mathbf{r}-\mathbf{r}^{\prime}\right|^{3}}\right]
$$

assuming that the sliding velocity is in the $\mathrm{x}$-direction, where $\mathbf{r}^{\prime}=v t \hat{x}+h \hat{z}$ is the location of the moving charge (where $\hat{x}$ and $\hat{z}$ are unit vectors in the $\mathrm{x}$ and 
z directions, respectively). In order to apply this result to a monolayer film of charge density $\rho(\mathbf{r})$, let us multiply Eq. (18) by $\rho(\mathbf{r})$ and integrate over volume to obtain $\mathbf{E}(\mathbf{r})$,

$$
\begin{aligned}
& \mathbf{E}(\mathbf{r})=(v / 2 \pi \sigma) \int d^{3} r^{\prime} \rho\left(\mathbf{r}^{\prime}\right)(4 \pi) \\
& \times(2 \pi)^{-3} \int d^{3} k\left(\mathbf{k} k_{x} / k^{2}\right) e^{i \mathbf{k} \cdot\left(\mathbf{r}-\mathbf{r}^{\prime}\right)}
\end{aligned}
$$

where we have written the field in Eq. (19) in terms of its Fourier transform. For a periodic monolayer film a height $\mathrm{h}$ above the surface of the substrate, $\rho(\mathbf{r})$ has the form

$$
\rho(\mathbf{r})=\delta(z-h) \sum_{\mathbf{G}} \rho_{\mathbf{G}} e^{i \mathbf{G} \cdot(\mathbf{r}-v t \hat{x})}
$$

where $\mathbf{G}$ denote the reciprocal lattice vectors of the film and $\rho_{\mathbf{G}}=A_{c}^{-1} \int_{u} d^{2} r \rho_{2}(\mathbf{r}) e^{-i \mathbf{G} \cdot \mathbf{r}}$, where the $\mathrm{u}$ on the integral sign signifies an integral over a unit cell of the film, $A_{c}$ is the unit cell area and $\rho_{2}(\mathbf{r})$ is the charge per unit area of the film. For simplicity, we are modeling the film by a collection of point charges. If the nonzero size of the charges in the film were taken into account, there would be form factors introduced in the summations over $\mathbf{G}$, which would fall off rapidly with increasing magnitude of $\mathbf{G}$. This can be approximately accounted for by including only 
the first one or two terms in the sums. Substituting Eq. (20) in Eq. (19) gives

$$
\mathbf{E}=2 v(2 \pi \sigma)^{-1} \sum_{\mathbf{G}} \rho_{\mathbf{G}} \int d k_{z}\left(\frac{\mathbf{k} G_{x}}{k_{z}^{2}+G^{2}}\right) e^{i \mathbf{k} \cdot\left[\mathbf{r}_{\|}-v t \hat{x}-(z+h) \hat{z}\right]},
$$

where $\mathbf{r}_{\|}$is the projection of $\mathbf{r}$ in the plane of the film, and where the $\mathrm{x}$ and $\mathrm{y}$ components of $\mathbf{k}$ are equal to the $\mathrm{x}$ and $\mathrm{y}$ components of film reciprocal lattice vectors. For components of $\mathbf{E}$ parallel to the substrate we obtain on performing the integral over $k_{z}$

$$
\mathbf{E}(\mathbf{r})=(v / \sigma) \sum_{\mathbf{G}} \rho_{\mathbf{G}}\left(\mathbf{G} G_{x} / G\right) e^{-G(z+h)} e^{i\left(\mathbf{G} \cdot \mathbf{r}+G_{x} v t\right)} .
$$

To find $\rho_{\mathbf{G}}$, we model the charge density of of each molecule by three charges as was done in the last section. Then, substituting $\rho(\mathbf{r})$ from Eq. (11) in the integral for $\rho_{\mathbf{G}}$ under Eq. (20), we obtain

$$
\rho_{\mathbf{G}}=-4 q A_{c}^{-1} \sum_{\beta} \sin ^{2}\left(\mathbf{G} \cdot \ell_{\beta} / 2\right) e^{i \mathbf{G} \cdot \rho_{\beta}} .
$$

Therefore,

$$
\begin{aligned}
& \mathbf{E}(\mathbf{r})=-v 4 q A_{c}^{-1} \sigma^{-1} \sum_{\beta, \mathbf{G}} \sin ^{2}(\mathbf{G} \cdot \ell / 2) \\
& \times\left(\mathbf{G} G_{x} / G\right) e^{-G(z+h)} e^{i\left[\mathbf{G} \cdot\left(\rho_{\beta}+\mathbf{r}\right)-G_{x} v t\right]},
\end{aligned}
$$

which reduces to an expression resembling Bruch's for the field in the small $\ell_{\beta}$ limit with the quadrupole moment of the molecule $\theta$ equal to $q \ell_{\beta}^{2}$, if we pretend 
that the system is not in the anomalous skin effect regime, and replace $\sigma_{\mathbf{G}}$ by $\sigma$ for the purpose of making a comparison. The force of friction acting on the film is given by

$$
\begin{gathered}
F=(1 / 2) R e \int d^{3} r \rho^{*}(\mathbf{r}) \mathbf{E}(\mathbf{r})=-\left(N \theta^{2} \mathbf{v} / \sigma\right) \\
\times A_{c}^{-1} \sum_{\mathbf{G}}\left|\sum_{\beta}\left(\mathbf{G} \cdot \hat{\ell}_{\beta}\right)^{2} e^{i \mathbf{G} \cdot \rho_{\beta}}\right|^{2}\left(\mathbf{G} G_{x} / G\right),
\end{gathered}
$$

where $\mathrm{N}$ is the number of molecules in the film, and thus the inverse slip-time $\tau^{-1}$ is given by

$$
\begin{gathered}
\tau^{-1}=(F / N M v)= \\
A_{c}^{-1}\left(\theta^{2} / M \sigma\right) \sum_{\mathbf{G}}\left|\sum_{\beta}\left(\mathbf{G} \cdot \hat{\ell}_{\beta}\right)^{2} e^{i \mathbf{G} \cdot \rho_{\beta}}\right|^{2}\left(G_{x}^{2} / G\right) e^{-2 G h},
\end{gathered}
$$

where $M$ is the mass of an adsorbed molecule and $\hat{\ell}_{\beta}$ is a unit vector along $\ell_{\beta}$. If as discussed above, we replace $\sigma_{\mathbf{G}}$ of section II by $\sigma$, Eq. (15) becomes identical to Eq. (26). This demonstrates the equivalence of Bruch's and Boyer's treatments.

\section{A Treatment of the Problem which Includes the Nonzero Width of the Surface Region}

Both Bruch's treatment of the problem and Boyer's are based on the classical model for a metallic surface, in which the electronic charge density drops to zero immediately on leaving the metal. In a quantum mechanical treatment, in 
contrast, the electronic charge density drops to zero over a distance of the order of a couple of Angstroms. The film almost certainly resides in a region just outside the bulk of the metal, in which the electronic charge density is nonzero (although it is decaying exponentially here). Ying, et. al., studied electron screening in the surface region using the Thomas-Fermi approximation[16]. They find that a charge placed at the surface will be screened, with a screening length not much longer than that in the bulk metal. The bulk metal Thomas Fermi expression for the screening charge density of a point charge can be used to model the charge density in the surface region analytically if one fits the screening length to that found in Ref. 16. The bulk Thomas expression for the screening charge density for a point charge is

$$
\begin{gathered}
\rho_{s}(\mathbf{r})=-(4 \pi)^{-1} q k_{s}^{2} e^{-k_{s} r} / r= \\
-q k_{s}^{2}(2 \pi)^{-3} \int d^{3} k \frac{1}{k^{2}+k_{s}^{2}} e^{i \mathbf{k} \cdot \mathbf{r}} .
\end{gathered}
$$

where $\mathrm{q}$ is the point charge whose screening is being considered and $k_{s}$ is the inverse Thomas Fermi screening length, which we will take here to be a parameter to be fit to Ying, et. al.'s calculations[16]. In Ref. 16, the quantity

$$
\int d x d y \rho(\mathbf{r})
$$


is plotted as a function of $\mathrm{z}$. The model of Eq. (27) gives for this quantity

$$
-(1 / 2) q k_{s} e^{-k_{s}|z|},
$$

which strongly resembles the quantity plotted in Fig. 1 of Ref. 16 . Then to fit the present approximate model to the results of Ref. 16, we can simply choose a value of $k_{s}$ for which Eq. (26) reproduces each of the plots in Fig. 1 of that reference. This method is rigorous when one can use the quasi-classical approximation, which is accurate if typical values of the screening length are much smaller than the thickness of the surface region. (See the appendix.) When this is not an appropriate limit, for example if the film is further out from the bulk of the metal, there is asymmetry in the screening charge density[17], but it is not such an extreme asymmetry as to invalidate using a spherically symmetric screening charge density, as is done here as a first approximation.

On the basis of this model, we conclude that when a film with charge density $\rho(\mathbf{r})$ moves along the surface of the metal with a velocity $\mathbf{v}$, the screening charge density given by

$$
\rho_{s}(\mathbf{r})=-(2 \pi)^{-3} k_{s}^{2} \int d^{3} r^{\prime} \int d^{3} k \frac{e^{i \mathbf{k} \cdot\left(\mathbf{r}-\mathbf{r}^{\prime}\right)}}{k^{2}+k_{s}^{2}} \rho\left(\mathbf{r}^{\prime}\right)
$$

moves with the same velocity resulting in a current density $\mathbf{J}_{\mathbf{s}}=\mathbf{v} \rho_{s}(\mathbf{r})$. Substi- 
tuting for the $\rho\left(\mathbf{r}^{\prime}\right)$ in terms of its Fourier transform, we obtain

$$
\begin{gathered}
\mathbf{J}_{\mathbf{S}}(\mathbf{r})=k_{s}^{2}(2 \pi)^{-1} \sum_{\mathbf{G}} \int d k_{z} \frac{e^{i \mathbf{G} \cdot \mathbf{r}} e^{i k_{z}(z-h)}}{G^{2}+k_{s}^{2}+k_{z}^{2}} \rho_{\mathbf{G}}= \\
(1 / 2) k_{s}^{2} \mathbf{v} A_{c}^{-1} \sum_{\mathbf{G}} e^{i \mathbf{G} \cdot \mathbf{r}} \frac{\exp \left[-\left(G^{2}+k_{s}^{2}\right)^{1 / 2}|z-h|\right]}{\left(G^{2}+k_{s}^{2}\right)^{1 / 2}} \rho_{\mathbf{G}} .
\end{gathered}
$$

The resulting Ohmic heating contribution to the force of friction can be found from

$$
F_{\text {fric }} v=\sigma^{-1} \int\left|\mathbf{J}_{\mathbf{s}}\right|^{2} d^{3} r
$$

Substituting Eq. (31) into Eq. (32) while replacing $\sigma$ by an effective conductivity $\sigma_{\mathbf{G}} \approx \sigma / G \ell$ to account for the anomalous skin effect [7] (like the effective conductivity used in section II) and placing it within the summation over $\mathbf{G}$, we obtain for the force of friction

$$
\mathbf{F}_{\text {fric }}=A k_{s}^{4} \mathbf{v} \sum_{\mathbf{G}} \sigma_{\mathbf{G}}^{-1}\left(G^{2}+k_{s}^{2}\right)^{-3 / 2}\left|\rho_{\mathbf{G}}\right|^{2}
$$

Then, we have for the inverse slip-time

$$
\begin{gathered}
\tau^{-1}=\frac{F_{\text {fric }}}{N M v}= \\
\frac{k_{s}^{4} \theta^{2}}{2 A_{c} M} \sum_{\mathbf{G}} \sigma_{\mathbf{G}}^{-1}\left(G^{2}+k_{s}^{2}\right)^{-3 / 2}\left|\sum_{\beta}\left(\mathbf{G} \cdot \hat{\ell}_{\beta}\right)^{2} e^{i \mathbf{G} \cdot \rho_{\beta}}\right|^{2}
\end{gathered}
$$

where we have substituted for $\rho_{\mathbf{G}}$ using Eq. (23), where A is the area of the film $A_{c}$ is the area of a unit cell and $\mathrm{N}$ is the number of unit cells in the film. For 
$k_{s} \approx 10^{8} \mathrm{~cm}^{-1}$ and $\theta \approx 10^{-26} \mathrm{esu}$, we obtain $\tau^{-1} \approx 10^{11} \mathrm{~s}^{-1}$, which is much larger than the electron-hole creation mechanism discussed earlier[13]. The quantity $\tau^{-1}$ found from Eq. (34) is about four orders of magnitude larger than $\tau^{-1}$ found from Eq. (15) because Eq. (34) does not contain the factor $e^{-2 G h}$, which appears in Eq. (22) (where $G=2 \AA^{-1}$ and $h=2 \AA$ ). If we use a value for $k_{s}$ a factor of 5 smaller (which is not unreasonable considering that we are in the surface region where $k_{s}$ could be noticeably smaller than its bulk value because the conduction electron density is smaller here), $\tau^{-1}$ becomes of order $10^{8} \mathrm{~s}^{-1}$, comparable to the experimental value[4].

Bruch's use of a classical (i.e., zero thickness) surface would be valid if the film resided well above the surface where the (classical) method of electrical images should be valid[17]. The treatment in this section assumes that the film resides in a part of the surface region in which the electron density is closer to its bulk value. The two treatments bracket the true situation, in which the film lies between these two extremes. Since both treatments give a large enough magnitude for $\tau^{-1}$ to account for the experimental results, one can say with confidence that the Ohm's law heating contribution to the friction is of sufficiently large magnitude to account for the experimental results of Ref. 4, as put forward in Refs. 1 and 2 . 


\section{Conclusions}

Bruch's calculation of the electronic friction acting on a film of nitrogen molecules harmonically oscillating on a metallic substrate, is re-done for the simpler case in which the film is sliding at constant speed. This treatment, which should give the same value for the slip-time as Bruch's treatment, clarifies Bruch's treatment and allows one to easily demonstrate that the force of friction found by calculating the force of the image charge acting back on the film and by calculating the Ohm's law heating inside the metallic substrate are equal. It also allows one to demonstrate the equivalence of Bruch's treatment with that due to Boyer of a charge sliding over a metallic surface. Since Boyer's treatment is equivalent to that used in Refs. 1 and 2, one is confident in saying that Bruch's treatment of the problem is an extension of these methods, which allows one to include the anomalous skin effect.

Persson has argued that the contribution to the friction due to the creation of electron-hole pairs should dominate over the Ohm's law heating mechanism by three orders of magnitude for a charged ion moving above the surface of a metallic substrate[13]. Since this mechanism depends on the density of normal electrons, which does not drop rapidly on falling below the superconducting transition temperature, it cannot account for the experimental result reported in Ref. 4. 
The calculation of the Ohm's law heating contribution of the friction presented in the previous sections, including the anomalous skin effect conductivity, as suggested by Bruch[5], however, can be of the same order of magnitude as the electron-hole contribution to the friction. Since the resistivity drops to zero over a relatively small temperature range on dropping below $T_{c}$, the latter mechanism for electronic friction drops rapidly in much the same way as in Ref. 4, and, as we have seen, it is large enough to explain the experimental observations.

\section{acknowledgments}

I wish to thank the Department of Energy for their financial support (Grant DE-FG02-96ER45585). I also wish to thank L. W. Bruch for discussions that I have had with him about his work on this problem, as well as R. Markiewicz and A. Widom.

\section{Appendix: Quasiclassical Treatment of the Screening of a Charge in}

\section{the Surface Region}

In the surface region, where the electron charge density is decreasing from its

bulk value down to zero, the wave functions in the jelleum model must take the form

$$
\psi_{\mathbf{k}}(\mathbf{r})=e^{i \mathbf{k} \cdot \mathbf{r}} f_{\mathbf{k}}(z),
$$


where $f_{\mathbf{k}}(z)$ drops from the value it has for $\mathrm{z}$ in the bulk region to zero for $\mathrm{z}$ well above the surface. Then the electron charge density is equal to

$$
\sum_{\mathbf{k}} n_{\mathbf{k}}\left|\psi_{\mathbf{k}}(\mathbf{r})\right|^{2}=\sum_{\mathbf{k}} n_{\mathbf{k}}\left|f_{\mathbf{k}}(z)\right|^{2}
$$

where $n_{\mathbf{k}}$ is the Fermi function, $\left(e^{(\epsilon(\mathbf{k})-\mu) / k_{B} T}+1\right)^{-1}$, where $\epsilon(\mathbf{k})$ is the electron energy of wavevector $\mathbf{k}$ and $\mu$ is the chemical potential. The potential $\phi(\mathbf{r})$ satisfies Poisson's equation

$$
\nabla^{2} \phi(\mathbf{r})=-4 \pi \sum_{\mathbf{k}} n_{\mathbf{k}}\left|f_{\mathbf{k}}(z)\right|^{2}
$$

Let us now consider the screening of a point charge $\mathrm{q}$ placed at a point $\mathbf{r}=z_{0} \hat{z}$ inside the surface region, where $\hat{z}$ is a unit vector in the $\mathrm{z}$-direction. In the linearized Thomas-Fermi treatment of screening, we assume that $\phi(\mathbf{r})$ is changed by a small amount $\delta \phi(\mathbf{r})$ because of the point charge, add this change in the potential to $\mu$ and linearize in $\delta \phi$. Carrying this out we find that $\delta \phi(\mathbf{r})$ satisfies

$$
\nabla^{2} \delta \phi(\mathbf{r})=-k_{s}^{2}(z) \delta \phi(\mathbf{r})+4 \pi \delta(\mathbf{r})
$$

where

$$
k_{s}^{2}(z) \approx 4 \pi \sum_{\mathbf{k}} \delta(\epsilon(\mathbf{k})-\mu)\left|f_{\mathbf{k}}(z)\right|^{2}
$$

in the low temperature limit. Taking the Fourier transform of Eq. (A4) with 
respect to the components of $\mathbf{r}$ parallel to the surface it becomes

$$
d^{2} \delta \phi\left(\mathbf{k}_{\|}, z\right) / d z^{2}=-\left(k_{s}^{2}(z)-k_{\|}^{2}\right) \delta \phi\left(\mathbf{k}_{\|}, z\right)+4 \pi \delta\left(z-z_{0}\right),
$$

where $\delta \phi\left(\mathbf{k}_{\|}, z\right)$ is the Fourier transform on $\delta \phi(\mathbf{r})$ over the coordinates parallel to the surface. Let us attempt to find a solution to Eq. (A6) of the form $\delta \phi=e^{-S(z)}$. Substituting in Eq. (A6) we obtain

$$
\left[(d S / d z)^{2}-d^{2} S / d z^{2}-\left(k_{s}^{2}(z)-k_{\|}^{2}\right)\right] e^{-S}=4 \pi \delta\left(z-z_{0}\right) .
$$

If typical values of the screening length are small compared to the width of the surface region, we can for most z neglect $d^{2} S / d z^{2}$ compared to $(d S / d z)^{2}$. With this approximation, we obtain a solution

$$
\delta \phi=\left(4 \pi q / 2 k_{s}^{\prime}\left(z_{0}\right) \exp \left[-\left|\int_{z_{0}}^{z} k_{s}^{\prime}\left(k^{\prime}\right) d z^{\prime}\right|\right]\right.
$$

where $\left(k_{s}^{\prime}\right)^{2}=k_{s}^{2}-k_{\|}^{2}$. In the extreme limit in which typical $k_{s}^{\prime-1}$ values are much smaller than the width of the surface region, we can replace the integral in the exponent by $k_{s}^{\prime}\left(z_{0}\right)\left(z-z_{0}\right)$ to a good approximation. The resulting form for $\delta \phi$ gives an inverse Fourier transform on $k_{\|}$proportional to

$$
\frac{e^{-k_{s}\left(z_{0}\right)\left|\mathbf{r}-z_{0} \hat{z}\right|}}{\left|\mathbf{r}-z_{0} \hat{z}\right|}
$$

the form of the result for Thomas-Fermi of a point charge in the bulk of the 
metal. While the extreme limit of screening length much smaller than the width of the surface region is not likely to occur, we do not expect the screening in real situations to be so qualitatively different than the bulk Thomas Fermi screening. For example, one would not expect the screening that occurs parallel to the surface to be qualitatively different than that normal to the surface, although there is certainly likely to be some anisotropy[17].

\section{References}

[1] Sokoloff J B, Tomassone M S and Widom A (2000) Phys. Rev. Lett. 84, 515.

[2] Novotny T and Velicky B (1999) Phys. Rev. Lett. 83, 4112.

[3] Popov V L (1999), Phys. Rev. Lett. 83, 1632.

[4] Dayo A, Alnasrallah W and Krim J (1998) Phys Rev.

Lett.

80, 1690; Renner R L, Rutledge J E and Taborek P (1999) Phys Rev Lett 83, 1261; Krim J (1999) Phys Rev Lett 83, 1262.

[5] Bruch L W (2000) Phys Rev B 61, 16201.

[6] Boyer T H (1974) Phys Rev A 9, 68.

[7] Pippard A B (1958) in Advances in Electronics and Electron Physics, ed L Marton (Academic Press, NY; Mattis D C and Bardeen J (1958), Phys Rev 111, 412. 
[8] Tomassone M S and Widom A (1997) Phys Rev B56, 4938.

[9] Persson B N J and Andersson S (1984) Phys Rev B 29, 1863.

[10] Persson B N J and Zaremba E (1984) Phys Rev B 30, 5669.

[11] Persson B N J and Zaremba E (1985) Phys Rev B 31, 1863.

[12] Persson B N J and Lang N (1982) Phys Rev B 26, 5409.

[13] Persson B N J and Tosatti E (1998) Surf Sci 411, 855; Persson B N J (2000)

Solid State Communications 115, 145.

[14] Reitz J R, Milford F J and Christy R W (1993) "Foundations of Electromagnetic Theory," 4th edition (Addison-Wesley, Reading Massachusetts), section $16-6$.

[15] The Angstrom scale periodic spatial variation of the field of the sliding film should not make Ohm's law heating inapplicable We can see this by making a Galilean transformation to a reference frame in which the film is at rest The Ohm's law heating comes about in this frame from the potential from the impurities in the substrate, which in this frame is time dependent since the impurities are moving, and thus can excite conduction electrons near the Fermi level. It is not prevented from doing so by the periodic potential of the film (parallel to the surface) because the periodic field cannot produce gaps which wipe out the entire Fermi surface. 
[16] Ying S C, Smith J R and Kohn W (1971) J Vac Sci and Tech 9, 575.

[17] Ying S C, Smith J R and Kohn, W (1973) Phys Rev B 11, 1483. 